\title{
Effect of high-frequency AC electromagnetic actuation on the dynamic of an excited cantilever beam
}

\author{
Amine Bichri, Mohamed Belhaq, Jarir Mahfoud ${ }^{1}$ \\ Laboratory of Mechanics, University Hassan II-Casablanca, Morocco \\ ${ }^{1}$ INSA-Lyon, LaMCoSUMR5259, Lyon, France
}

\begin{abstract}
The effect of high-frequency AC electromagnetic actuation (EMA) on the dynamic behavior of a harmonically excited cantilever beam is analyzed in this paper. Analytical treatment based on perturbation analysis is performed on a simplified one degree of freedom equation modelling the first bending mode of the cantilever beam. The results show that under a certain specific condition relating the intensity of the fast AC to the displacement, the nonlinear characteristic of the system can be controled.
\end{abstract}

\section{Introduction}

Electromagnetic actuators (EMAs) are simple and reliable means of vibration excitation that are used in many industrial applications where attracting forces are needed. The generated force, proportional to the square of the current and inversely proportional to the square of the length of the air gap, can be exploited positively for controlling nonrotating structure. Basically, EMAs are used in many engineering applications, such as automotive start motors, electric door, hydraulic valves, power relays, and others; see for instance [1-4].

The systems actuated by electromagnetic forces exhibit in general complicated behavior due to the nonlinearities generated by the force. For instance, EMA can produce nonlinear dynamic and chaotic response [5]. In a recent study, an active electromagnetic damping of lateral vibration of a clamped steel cantilever has been investigated in [6].

In [7] an EMA was proposed for controlling the vibration of a cantilever beam with a tip mass and it was shown numerically and experimentally possibilities of suppressing vibration of the beam. Halmai and Lukács [8] designed and manufactured a new linear-electromagnetic actuator for cellular phones using metal springs. Der Hagopian and Mahfoud [9] designed and characterized experimentally EMA. They evaluated numerically and experimentally the possibility of controlling a flexible beam.

In a recent work [10], the effect of EMAs on the dynamic of a periodically excited cantilever beam in a single mode approximation was investigated analytically, numerically and using experimental testing. It was shown that the force induced by the EMAs introduces a softening behavior into the system and causes the resonance curve to shift left. Further, it was shown that as the intensity of the current of the EMAs is increased, the softening characteristic of the system increases.

The objective of this paper is to study analytically, and numerically, the influence of EMAs on the frequency response of an excited cantilever beam in a single mode approximation. Specifically, we analyze the effect of varying the intensity of the current generating the EMAs force on the dynamic behavior of the system. We perform an analytical investigation on a one degree of freedom system consisting in a nonlinear oscillator subjected to a periodic excitation. The analytical treatment based on a perturbation analysis leads to an approximation of the amplitudefrequency response equation allowing the analysis of the influence of EMA on the response.

\section{Equation of motion and slow dynamic}

The equation of motion that describes the dynamic behavior of a cantilever beam in a single mode approximation subjected to a harmonic external excitation and to electromagnetic forces, $F_{e m}$, can be written as [10]

$$
m \ddot{\delta}+\alpha \dot{\delta}+k \delta=F \cos \omega t+F_{e m}
$$

where $m$ is the mass, $\alpha$ is the damping coefficient, $k$ is the stiffness, $F$ and $\omega$ are the amplitude and the frequency of the external forcing, respectively. The EMAs are supplied by a constant current generating a static force $F_{D C}$ and a high-frequency actuation generating a fast alternative force $F_{\text {AC }}$ such that

$$
F_{e m}=F_{A C}+F_{D C}
$$

where $\quad F_{A C}=\frac{C_{1} i_{1}^{2}}{(\lambda-\delta)^{2}}-\frac{C_{1} i_{2}^{2}}{(\lambda+\delta)^{2}} \quad$ and $F_{D C}=\frac{C_{0} I_{0}^{2}}{(\lambda-\delta)^{2}}$.

Here the parameters $\lambda, C_{0}, C_{1}$ depend on the geometrical characteristics of the actuators and $\delta$ is the colocolized displacement. The current $i_{1}$ is given by $i_{1}=I_{1} \cos \kappa t$ where $I_{1}$ and $\kappa$ are, respectively, the amplitude and the frequency of the modulated current.

Assume that during the operation the currents $I_{1}$ and $i_{2}$ satisfy the relation

$$
i_{2}=\frac{I_{1}(\lambda+\delta)}{\sqrt{2}(\lambda-\delta)}
$$

relating the intensity of the alternative current $I_{1}$ and $i_{2}$ to the displacement. Under this condition, the total electro- 
magnetic force $F_{e m}$ reads

$$
F_{e m}=\frac{C_{0} I_{0}^{2}}{(\lambda-\delta)^{2}}+\frac{C_{1} I_{1}^{2}}{2(\lambda-\delta)^{2}} \cos 2 \kappa t
$$

Substituting Eq. (4) into Eq. (1), we obtain the dimensionless equation of motion

$$
z^{\prime \prime}+c z^{\prime}+z=f \cos v t+\frac{a_{0}}{(1-z)^{2}}+\frac{a_{1}}{(1-z)^{2}} \cos \Omega \tau
$$

where $z=\frac{\delta}{\lambda}, c=\frac{\alpha}{m \omega_{0}}, \omega_{0}^{2}=\frac{k}{m}, v=\frac{\omega}{\omega_{0}}, \Omega=\frac{2 \kappa}{\omega_{0}}, f=\frac{F}{\lambda m \omega_{0}^{2}}$, $a_{0}=\frac{C_{0} I_{0}^{2}}{\lambda^{3} m \omega_{0}^{2}}, a_{1}=\frac{C_{1} I_{1}^{2}}{2 \lambda^{3} m \omega_{0}^{2}}$ and $\tau=\omega_{0} t$.

The primes in Eq. (5) represent differentiation with respect to the time $\tau$. In the case under consideration, the vibrations of the cantilever beam are assumed to have small amplitudes around the trivial equilibrium. In this case, it is convenient to use Taylor series expansion of the nonlinear terms in the r.h.s. of Eq. (5) keeping only terms up to order three in $z$. Thus, the equation of motion takes the form

$$
\begin{array}{r}
z^{\prime \prime}+c z^{\prime}+z\left(1-2 a_{0}\right)-3 a_{0} z^{2}-4 a_{0} z^{3}-a_{0}= \\
f \cos v \tau+a_{1}\left(1+2 z+3 z^{2}+4 z^{3}\right) \cos \Omega \tau
\end{array}
$$

To derive the slow dynamic of system (6), we use the method of direct partition of motion [12] by introducing two different time scales, a fast time $T_{0}=\Omega \tau$ and a slow time $T_{1}=\tau$, and we split up $z(\tau)$ into a slow part $x\left(T_{1}\right)$ and a fast part $\phi\left(T_{0}, T_{1}\right)$ as follows

$$
z(\tau)=x\left(T_{1}\right)+\epsilon \phi\left(T_{0}, T_{1}\right)
$$

where $x$ describes the slow main motions at time-scale of oscillations and $\phi$ stands for an overlay of the fast motions. The frequency $\Omega$ is considered as a large parameter, for convenience. Using averaging method leads to the approximate equation for the slow motion

$$
x^{\prime \prime}+c x^{\prime}+\Omega_{0}^{2} x+\beta x^{2}-\gamma x^{3}+G=f \cos v \tau
$$

where $\Omega_{0}^{2}=1-2 a_{0}+\frac{5 a_{1}^{2}}{\Omega^{2}}, \beta=\frac{15 a_{1}^{2}}{\Omega^{2}}-3 a_{0}, G=\frac{a_{1}^{2}}{\Omega^{2}}-a_{0}$ and $\gamma=4 a_{0}-\frac{25 a_{1}^{2}}{\Omega^{2}}$.

The dimensionless force induced by the equivalent linear and nonlinear stiffness is given by

$$
F(x)=\Omega_{0}^{2} x+\beta x^{2}-\gamma x^{3}+G
$$

Figure 1a shows the variation of the effective force in the absence of DC showing that the behavior of the beam becomes hardening by increasing AC.

Figure $1 \mathrm{~b}$ shows that the presence of DC and AC actuations may changes the behavior of the beam from softening to hardening by increasing the AC.

\section{Frequency response of the slow dynamic}

The analysis of the slow dynamic near the primary resonance can be carried out using the multiple scales technique [11]. By expressing the resonance condition according to

$$
\Omega_{0}^{2}=v^{2}+\sigma
$$
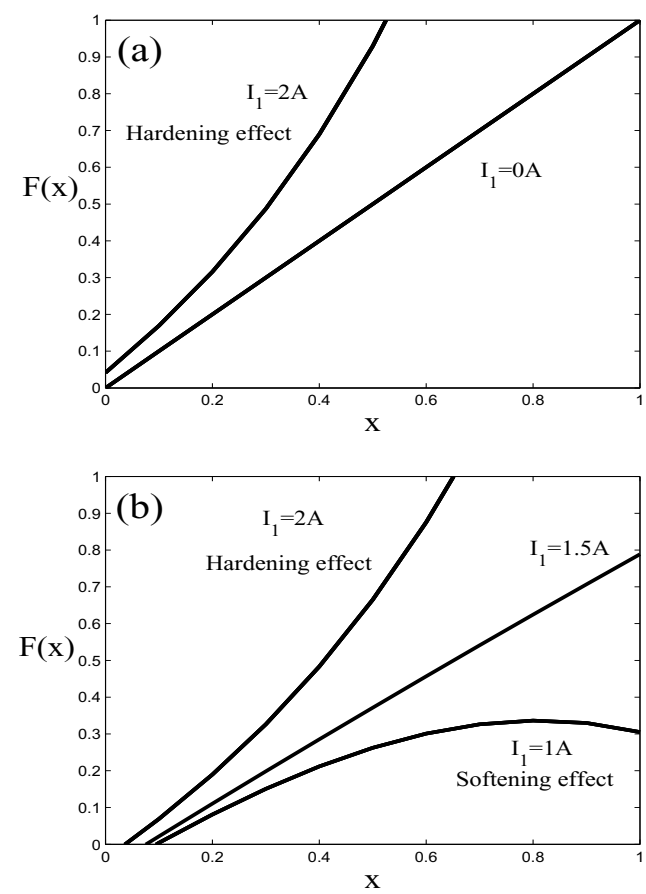

Fig. 1. Variation of the dimensionless force induced by the equivalent stiffness versus the displacement for $\Omega=5, m=1 \mathrm{Kg}$, $K=6.67 \times 10^{3} \mathrm{~N} / \mathrm{m}, C_{1}=C_{0}=4.27 \times 10^{-27} \mathrm{Nm}^{2} A^{-2}, \lambda=1.1 \mathrm{~mm}$; (a) $I_{0}=0 \mathrm{~A}$, (b) $I_{0}=0.4 \mathrm{~A}$.

where $\sigma$ is the detuning from the resonance and introducing a book-keeping parameter $\eta$ in the nonlinear component, Eq. (8) reads

$$
x^{\prime \prime}+v^{2} x=\eta\left[f \cos v \tau-c x^{\prime}-\sigma x-\beta x^{2}-G\right]+\eta^{2} \gamma x^{3}
$$

Performing the multiple scales method, we obtain the equation of amplitude and phase

$$
\left\{\begin{array}{l}
\frac{d r}{d \tau}=A r+H_{1} \sin \theta+H_{2} \cos \theta \\
r \frac{d \theta}{d \tau}=B r+C_{n l} r^{3}+H_{1} \cos \theta-H_{2} \sin \theta
\end{array}\right.
$$

The effective nonlinearity coefficient $C_{n l}=-\left(\frac{3 \gamma}{8 v}+\frac{5 \beta^{2}}{12 v^{3}}\right)$ and $A=\frac{-c}{2}, B=\frac{\sigma}{2 v}-\frac{c^{2}}{8 v}-\frac{\sigma^{2}}{8 v^{3}}-\frac{\beta G}{v^{3}}, H_{1}=\left(\frac{f \sigma}{8 v^{3}}-\frac{f}{2 v}\right), H_{2}=\frac{f c}{8 v^{2}}$. By eliminating the phase $\theta$ from the system (12), one obtains the amplitude-frequency response equation

$$
C_{n l}^{2} r^{6}+2 B C_{n l} r^{4}+\left(A^{2}+B^{2}\right) r^{2}-\left(H_{1}^{2}+H_{2}^{2}\right)=0
$$

In Fig. 2 we plot the effective nonlinear coefficient $C_{n l}$ versus $I_{1}$. This curve indicates that the effective nonlinearity vanishes and changes sign for the critical value of the AC, $I_{1} \simeq 1.5 \mathrm{~A}$. In this case the solution of Eq. (13) is given by

$$
r=\sqrt{\frac{H_{1}^{2}+H_{2}^{2}}{A^{2}+B^{2}}}
$$

Figure 3 shows the frequency-response curves, as given by Eq. (13), for given values of the intensity $I_{0}$ and the frequency $\Omega$ and for different values of the intensity of the alternating current $I_{1}$. 


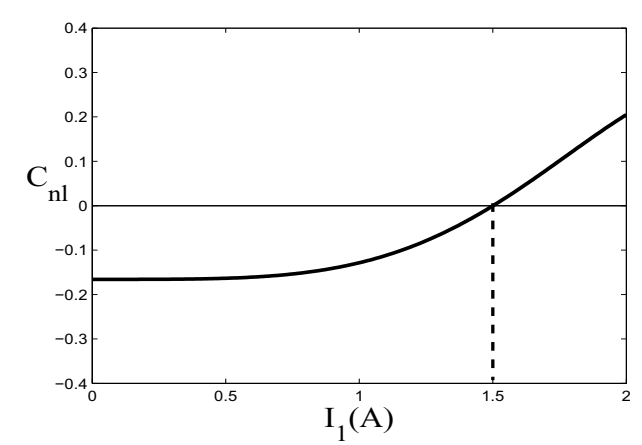

Fig. 2. Variation of the effective nonlinear coefficients with $I_{1}$ for $\Omega=5, v=\Omega_{0}$ and $I_{0}=0.4 A$.
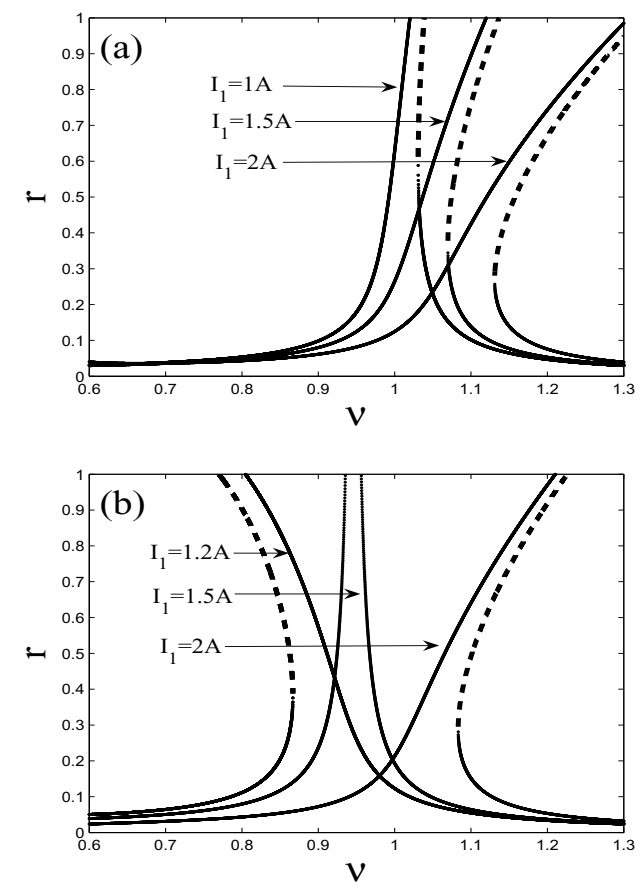

Fig. 3. Amplitude-frequency response near the primary resonance for $\Omega=5, c=0.005, f=0.02$; (a) $I_{0}=0 A$, (b) $I_{0}=0.4 \mathrm{~A}$.

Figure $3 \mathrm{a}$ shows the frequency-response curves in the absence of the DC $\left(I_{0}=0\right)$. It can be seen that in this case the increasing of the intensity of the $\mathrm{AC}$ hardens the system. Figure $3 \mathrm{~b}$ indicates that for the critical value of the $\mathrm{AC}$, $I_{1 c} \simeq 1.5 A\left(C_{n l}=0\right)$, the system has a linear behavior. For values of $I_{1}<I_{1 c}\left(C_{n l}<0\right)$ the frequency-response curve shows a softening behavior, while for values of $I_{1}>I_{1 c}$ $\left(C_{n l}>0\right)$ the response curve indicates a hardening behavior.

\section{Conclusion}

We have studied the effect of the intensity of a high frequency $\mathrm{AC}$ of an EMA on the dynamic of an excited cantilever beam modeled by its first bending mode and presented by a single degree of freedom oscillator. Attention was focused on the dynamic near primary resonance and the approach was performed using perturbation analysis to approximate the frequency response curve. The results shown that depending on the amplitude of the alternative current of the EMA, the frequency response of the first bending mode of the cantilever beam can be shifted towards lower or higher values of frequencies producing softening or hardening behavior in the system. This result indicates that it is possible to control the nonlinear characteristic of the response near the resonance by tuning the intensity of the AC.

\section{References}

1. Schweitzer G., Bleuler H., Traxler A., Active magnetic bearings - basics, properties and applications, vdf Hochschulverlag AG, ETH, Zurich, (2003).

2. El-Shafei A., Dimitri A.S., Controlling journal bearing instability using active magnetic bearings, Proceedings of ASME Turbo Expo, GT-28059, Canada (2007).

3. Skladanek Y., Der Hagopian J., Mahfoud J., Energy cost of the active control of rotating machine by using electromagnetic actuator and piezoelectric actuator, ASME Gas Turbine Technical Congress Exposition, June 8-12, Orlando, Florida, USA (2009).

4. Gutman I., Industrial uses of mechanical vibrations, business books limited, London, (1968).

5. Chang S.C., Ling H.P., Nonlinear dynamics and chaos control for an electromagnetic system, Journal of Sound and Vibration. 279, (2005) 327-344.

6. Gospodaric B., Voncina D., Bucar B., Active electromagnetic damping of laterally vibrating ferromagnetic cantilever beam, Mechatronics. 17, (2007) 291-298.

7. Der Hagopian J., Mahfoud J., Numerical and experimental investigation of the active control by using electromagnetic actuator, Proceedings of the XIII International Symposium on Dynamic Problems of Mechanics, Almeida, C. A. (Editor), ABCM, Angra dos Reis, RJ, Brazil, (2009).

8. Halmai A., Lukács A., New linear-electromagnetic actuator used for cellular phones, Periodica Polytechnica, Mechanical Engineering. 51, (2007) 19-22.

9. Der Hagopian J., Mahfoud J., Electromagnetic actuator design for the control of light structures, Smart Structures and Systems. 6, (2010) 29-38.

10. Belhaq M., Bichri A., Der Hogapian J., Mahfoud J., Effect of electromagnetic actuations on the dynamics of a harmonically excited cantilever beam, International Journal of Non-Linear Mechanics. 46, (2011) 828-838.

11. Nayfeh A.H., Mook D.T., Non-linear Oscillations. Wiley, New York, (1979).

12. Blekhman I.I., Vibrational Mechanics - Nonlinear Dynamic Effects, General Approach, Application, Singapore: World Scientific (2000). 